\title{
Durable staged hybrid ablation with thoracoscopic and percutaneous approach for treatment of long-standing atrial fibrillation: A 30-month assessment with continuous monitoring
}

\author{
Claudio Muneretto, MD, ${ }^{\mathrm{a}}$ Gianluigi Bisleri, MD, ${ }^{\mathrm{a}}$ Luca Bontempi, MD, ${ }^{\mathrm{b}}$ and Antonio Curnis, $\mathrm{MD}^{\mathrm{b}}$
}

\begin{abstract}
Objectives: Electrophysiologic and surgical procedures to treat stand-alone atrial fibrillation (AF) have recently evolved, but disappointing results in patients with long-standing persistent (LSP) AF have challenged the durability of these procedures.
\end{abstract}

\begin{abstract}
Methods: Lone AF patients $(n=36)$ with either LSP-AF $(n=28)$ or persistent AF $(n=8)$ were prospectively enrolled in the study and consecutively treated by thoracoscopic ablation followed by electrophysiologic evaluation 30 days afterward. Mean left atrial dimension was $50.3 \pm 5.5 \mathrm{~mm}$, and average AF duration was 72.8 months (range, 7-240 months). The thoracoscopic procedure was a right monolateral approach to create a box lesion using a temperature-controlled radiofrequency device with suction adherence. A continuous rhythm monitoring device was implanted at the end of the operation.
\end{abstract}

Results: Thoracoscopic ablation was successfully completed without morbidity or mortality and without any intensive care unit stay. Intraoperative exit and entrance block was achieved in $100 \%$ and $88.8 \%(32 / 36)$ of patients, respectively. At $33 \pm 2$ days after the operation, an electrophysiologic study confirmed entry-exit block in $83.3 \%$ (30/36) whereas pulmonary vein reconnections were observed in $16.7 \%(6 / 36)$ of patients. Additional transcatheter lesions were performed in $61.1 \%$ (22/36) of patients. At a mean follow-up of 30 months (range, 1-58 months), $91.6 \%(33 / 36)$ of patients are in sinus rhythm with $77.7 \%(28 / 36)$ of these patients off antiarrhythmic drugs and $88.8 \%$ (32/36) free of warfarin. Long-term incidence of left atrial flutter was $0 \%$.

Conclusions: The combination of a surgical box lesion and transcatheter ablation in a hybrid approach provided excellent durable clinical outcomes in patients with LSP-AF. (J Thorac Cardiovasc Surg 2012;144:1460-5)

Surgical and percutaneous approaches have been aiming at effectively treating atrial fibrillation $(\mathrm{AF})$, with progressive technical and technologic refinements from either side occurring during the past decade. From the surgical standpoint, several less-invasive procedures have been steadily adopted albeit with a limited set of lesions and less favorable results when compared with the Cox maze procedure. $^{1-4}$ Electrophysiologists (EP) have dramatically evolved as well from the original description of focal pulmonary vein (PV) triggers of ablation, albeit with poorer success rates than surgical ablations and with the need for multiple procedures, especially in the presence of persistent $\mathrm{AF}$ (P-AF) and long-standing persistent $\mathrm{AF}$ (LSP-AF). ${ }^{5,6}$

From the Divisions of Cardiac Surgery ${ }^{\mathrm{a}}$ and Cardiology, ${ }^{\mathrm{b}}$ University of Brescia Medical School, Brescia, Italy.

Disclosures: Drs Muneretto and Bisleri are consultants for Estech. The other authors have nothing to disclose with regard to commercial support.

Read at the 92nd Annual Meeting of The American Association for Thoracic Surgery, San Francisco, California, April 28-May 2, 2012.

Received for publication April 21, 2012; revisions received Aug 21, 2012; accepted for publication Aug 24, 2012; available ahead of print Oct 15, 2012.

Address for reprints: Claudio Muneretto, MD, Cardiochirurgia SSVD—Spedali Civili, P.le Spedali Civili, 1, 25123 Brescia, Italy (E-mail: munerett@med. unibs.it).

0022-5223/\$36.00

Copyright (C) 2012 by The American Association for Thoracic Surgery

http://dx.doi.org/10.1016/j.jtcvs.2012.08.069
In particular, there has been an increasing awareness that PV isolation alone could not suffice for an effective treatment of AF, especially in this specific subset of patients: surgical ablation per se could potentially offer an extensive and effective PV and left atrial isolation, while EP approaches could allow for tailored additional lesions to achieve durable results even in LSP-AF. ${ }^{7}$

We therefore aimed at combining a minimally invasive surgical and catheter-based approach to merge respective advantages and to reduce drawbacks of each procedure in a sequential, staged hybrid fashion.

\section{METHODS}

\section{Study Population}

From November 2006 to September 2011, 36 consecutive patients with either P-AF $(\mathrm{n}=8,22.2 \%)$ or LSP-AF $(\mathrm{n}=28,77.8 \%)$ were prospectively enrolled. As per Heart Rhythm Society (HRS) recommendations ${ }^{8}$ inclusion criteria for stand-alone AF ablation were patients with P-AF/LSP-AF with refractoriness to at least 1 antiarrhythmic drug (either class I or III) and without any concomitant heart disease. Exclusion criteria were previous cardiac surgery, previous catheter ablation for $\mathrm{AF}$ treatment, and left atrial-anteroposterior diameter greater than $60 \mathrm{~mm}$. A detailed breakdown of patient characteristics is outlined in Table 1; in particular, mean left atrial-anteroposterior dimension was $50.3 \pm 5.5 \mathrm{~mm}$, and average AF duration was 72.8 months (range, 7-240 months).

As per the study protocol, patients underwent first an endoscopic, epicardial surgical ablation with intraoperative assessment of the lesion set 


\section{Abbreviations and Acronyms \\ $\mathrm{AF} \quad=$ atrial fibrillation \\ EP = electrophysiologic (electrophysiologist) \\ HRS = Heart Rhythm Society \\ LSP-AF $=$ long-standing persistent atrial fibrillation \\ $\mathrm{P}-\mathrm{AF}=$ persistent atrial fibrillation \\ $\mathrm{PV} \quad=$ pulmonary vein}

followed by an EP evaluation and potential "touch-up" between 30 and 45 days postoperatively in a sequential, staged fashion.

The study protocol was approved by the Institutional Review Board and Ethical Committee (No. NP1076) of the University of Brescia Medical School.

\section{First Step-Surgical Procedure}

All patients underwent a routine preoperative evaluation, including 3-dimensional computed tomographic scan reconstruction, to obtain a detailed preoperative plan, in particular with respect to PV anatomy and variations.

The surgical ablation consisted of a closed-chest, right-sided monolateral approach to deliver a continuous lesion encircling the origin of all PVs and the posterior aspect of the left atrium en bloc ("box" lesion set) as previously described. ${ }^{9}$ In particular, care was taken during pericardial opening to stay at least 2.5 to $3 \mathrm{~cm}$ away from the phrenic nerve so as to minimize the incidence of postoperative phrenic nerve palsy and right diaphragm elevation. Of note, an extensive removal of the epicardial fat, especially at the level of the Waterston groove and the roof of the left atrium, was performed before energy delivery in all patients. In fact, this maneuver yields benefits both in terms of removal of ganglionic plexi and as better adherence of the ablation probe with the atrial tissue and therefore improved energy penetration. The ablation device used is a temperaturecontrolled, monopolar radiofrequency device with suction adherence and internal cooling (Cobra Adhere XL; Estech, San Ramon, Calif). The Cobra Adhere $\mathrm{XL}$ is an ablation probe with CE mark and US investigational device exemption investigation (April 2012). The device is set at a maximum power limit of 150 watts with a temperature control limit of $80^{\circ} \mathrm{C}$. A proper adherence of the probe with the epicardial tissue occurs at values around $500 \mathrm{~mm} \mathrm{Hg}$ of negative pressure; then, energy is delivered and ablation continued for a 120 -second ablation time. All electrodes (10 segments, 5 proximal and 5 distal) of the Cobra Adhere XL must be activated to allow for continuous box lesions. Either the proximal (at the level of the transverse sinus down to the left atrial appendage) or the distal ones (at the level of the oblique sinus) are sequentially activated. It is of utmost importance to deliver multiple ablations (at least twice per proximal or distal segment) on the epicardial surface to achieve an effective isolation. Moreover, an extensive overlapping is performed at the level of the Waterston groove to ensure the "closure" of the box lesion.

At the completion of the surgical ablation, intraoperative assessment is performed according to conventional EP parameters to demonstrate entrance and/or exit block across the box lesion, as per HRS recommendations. ${ }^{8}$ The PVs were monitored for reconduction 20 minutes after the initial PV isolation. The study protocol mandated the achievement at least of unidirectional block through the box lesion; if this end point was unmet, additional ablations were delivered until either entry or entry block (or both) were obtained.

The protocol outlined was used in all patients of the current series. A $6 \mathrm{~F}$ decapolar electrode endocardial catheter (P-Supra CS; Biosense Webster, Diamond Bar, Calif) that has been previously positioned in the EP laboratory at the level of the coronary sinus is connected to an EP workstation.
A tetrapolar EP catheter (Avail, Josephson Curve, type A; Biosense Webster) is then introduced through a port and advanced epicardially within the box lesion (at the level of the right PVs and the roof of the left atrium) and connected as well to the EP workstation. As depicted in Figures 1, A, and 2, $A$, the catheter within the box lesion is used as a pacing probe while sensing with the catheter in the coronary sinus (exit block) or vice versa (entrance block). In either instance, the maximum pacing output is $20 \mathrm{~mA}$.

At the end of the surgical procedure, an implantable loop recorder (Reveal XT, Medtronic, Inc, Minneapolis, Minn) is implanted in all patients.

\section{Postoperative Management}

Postoperative pharmacologic management included the following:

Antiarrhythmic protocol. The antiarrhythmic protocol was mostly based on amiodarone intake (or sotalol in case of contraindication for amiodarone intake) for at least 3 months after the procedure.

Anticoagulation protocol. The anticoagulation protocol was maintained for at least 3 months after the procedure. In case of stable sinus rhythm, after an echocardiographic evaluation to rule out potential atrial stasis or thrombus in the left atrium, anticoagulation was withdrawn and aspirin started.

Moreover, given the potential inflammatory subset after ablation, ${ }^{10}$ all patients received steroid intake after the first (surgical) step by means of prednisone $25 \mathrm{mg}$ per day for $1 \mathrm{week}$, then tapered to $12.5 \mathrm{mg}$ for the second week, finally to $6.25 \mathrm{mg}$ for the third week, and then withdrawn.

After the surgical ablation, a blanking period of 3 months was established before rhythm outcome was analyzed. Rhythm outcome was assessed by data analysis of the Reveal XT on a monthly basis to rule out potential AF recurrences and relative burden. Stable sinus rhythm was considered as the absence of AF episodes lasting more than 5 minutes and an overall burden of $0.5 \%$ of time spent in $\mathrm{AF}$ on a monthly basis, as previously reported. ${ }^{11,12}$ Data were obtained via the CareLink Network (Medtronic, Inc), which allows for online storage and retrieval of data without the need for an outpatient visit.

\section{Second Step-EP Evaluation}

The study protocol mandated that all patients undergo a staged catheter procedure 30 to 45 days after the surgical ablation to assess the durability of conduction block and to deliver additional ablation lesions via a transcatheter route if required. At a minimum, the treatment protocol required that any gaps in the box lesion be closed by endocardial ablation. In all cases the Cartomerge (Biosense Webster) system was used for electroanatomic reconstruction. Noninducibility of $\mathrm{AF}$ and atrial flutter was also evaluated by atrial burst pacing.

\section{RESULTS}

\section{First Step-Surgical Procedure}

The closed-chest surgical ablation was successfully completed in all patients without any intraoperative complications. Mean ablation and overall procedural times were $31 \pm 7$ minutes and $80 \pm 7$ minutes, respectively. Ablation was performed as previously outlined in the Methods section. At a minimum, unidirectional block had to be confirmed; documented intraoperative exit block was achieved in $100 \%$ of patients whereas entrance block was achieved in $88.8 \%(32 / 36)$ of patients.

No patients required a stay in the intensive care unit, and no complications occurred during the postoperative stay (mean duration, $4 \pm 1.8$ days). In particular, the incidence of late coronary events (especially to the circumflex artery) 
TABLE 1. Preoperative characteristics

\begin{tabular}{lc}
\hline Age, y (mean \pm SD) & $62.3 \pm 10$ \\
Female gender, $\mathrm{n}(\%)$ & $19(52.7 \%)$ \\
Diabetes mellitus, $\mathrm{n}(\%)$ & $7(19.4 \%)$ \\
Hypertension, $\mathrm{n}(\%)$ & $15(41.6 \%)$ \\
No. of failed AADs & $1.9 \pm 0.4$ \\
AF type & \\
$\quad$ Persistent, n (\%) & $8(22.2 \%)$ \\
$\quad$ Long-standing persistent, $\mathrm{n}(\%)$ & $28(77.8 \%)$ \\
AF duration, mo (mean and range) & $72.8(7-240)$ \\
LA size, mm (mean \pm SD) & $50.3 \pm 5.5$ \\
LVEF, \% (mean \pm SD) & $52.5 \pm 3.3$ \\
\hline
\end{tabular}

$A A D s$, Antiarrhythmic drugs; $A F$, atrial fibrillation; $L A$, left atrial; $S D$, standard deviation; $L V E F$, left ventricular ejection fraction.

or right hemidiaphragm elevation was $0 \%$. Hospital mortality was $0 \%$.

\section{Second Step-EP Evaluation}

After a postoperative interval of $33 \pm 2$ days, all patients underwent an EP evaluation: bidirectional (entrance-exit block) was confirmed in $83.3 \%(30 / 36)$ of patients with PV reconnections observed in $16.7 \%(6 / 36)$, which were obviously treated percutaneously. As previously outlined, noninducibility of AF and atrial flutter was evaluated by atrial burst pacing.

Overall, transcatheter lesions were performed in $61.1 \%$ (22/36) of the patients. Besides gaps in the box lesion (6/36 patients, $16.7 \%$ ), additional ablations were mostly targeted at the cavotricuspid isthmus (owing to inducible or a positive history of typical flutter) in $58.3 \%(21 / 36)$ of the patients, whereas complex atrial fractionated electrograms were targeted at $16.6 \%(6 / 36)$ of patients. Finally, in $38.8 \%$ (14/36) of patients, firing foci within the PVs could be identified during the EP evaluation, and an additional PV antral line was performed even in the presence of an effective box ablation line (with block confirmation), so as to provide an additional "barrier" in case of potential later $\mathrm{PV}$ reconnection.

In case of box lesion set evaluation only (transseptal puncture and lesion test), mean procedural time was $18 \pm 2$ minutes, and additional ablations time was $25 \pm 4$ minutes.

No atypical left-sided flutter could be induced and therefore no connecting lesion to the mitral anulus was performed.

\section{Midterm Follow-up}

Patients were observed for a mean follow-up of 30 months (range, 1-58 months; median, 28 months) and rhythm outcomes were monitored on a monthly basis via the Reveal XT and CareLink Network. Of note, only 1 patient had a postoperative follow-up of 1 month, whereas all other patients in the series had been evaluated for at least 8 months. Stable sinus rhythm was considered as the absence of AF episodes lasting more than 5 minutes and an overall burden of $0.5 \%$ of time spent in AF on a monthly basis, as previously outlined ${ }^{11,12} ; 91.6 \%(33 / 36)$ of patients are in sinus rhythm with $77.7 \%(28 / 36)$ of these patients off antiarrhythmic drugs and $88.8 \%$ (32/36) free from anticoagulation (after echocardiographic evaluation to rule out potential atrial stasis or thrombus in the left atium). Of note, the long-term incidence of left atrial flutter was $0 \%$.

\section{DISCUSSION}

The challenges in the treatment of P-AF have demonstrated the drawbacks of both the surgical and the percutaneous approach. From the surgical standpoint, the invasiveness of the Cox maze procedure has hampered its wide adoption, despite the relevant success rates; transcatheter procedures instead are less-invasive procedures that are, however, associated with poor outcomes even after several ablations at long-term follow-up, especially in the presence of LSP-AF. ${ }^{5}$

The reason to merge surgical and percutaneous techniques and technologies in a hybrid fashion is therefore to reduce the complications and drawbacks of either procedure while improving the results of both approaches.

When a hybrid approach is used, it can be performed as either a concomitant or staged procedure, as previously described. ${ }^{713-15}$ We decided to perform surgery first inasmuch as it allows for an extensive isolation of the PV and the posterior aspect of the left atrium, thereby excluding not only the potential focal triggers located within the PVs but also the macroreentrant circuits and the fragmented potential, which are usually located in this anatomic region. From the EP perspective, such surgical radiofrequency ablation yields considerable advantages since the recent HRS consensus statement also recommends that radiofrequeny power be reduced when treating the posterior left atrium to reduce the risk of potential collateral damage to the esophagus. Therefore, this procedural limitation could represent a drawback of transcatheter ablation and potentially jeopardize success. Instead, such risk can be mitigated by the surgical box lesion set.

Moreover, from the surgical standpoint, if deemed necessary it is possible to easily create an additional intercaval lesion (from the superior to the inferior vena cava) epicardially, thereby entirely avoiding the risk associated with phrenic nerve palsy after a transcatheter approach, which is estimated to occur in around $6 \%$ of cases. ${ }^{16}$

As previously outlined in the Methods section, assessment of PV anatomy is of utmost importance from both the surgical and the EP perspectives. Before the surgical procedure, any relevant anatomic variation can be ruled out because of the presence of an additional right inferior PV that may inadvertently be damaged during the opening 


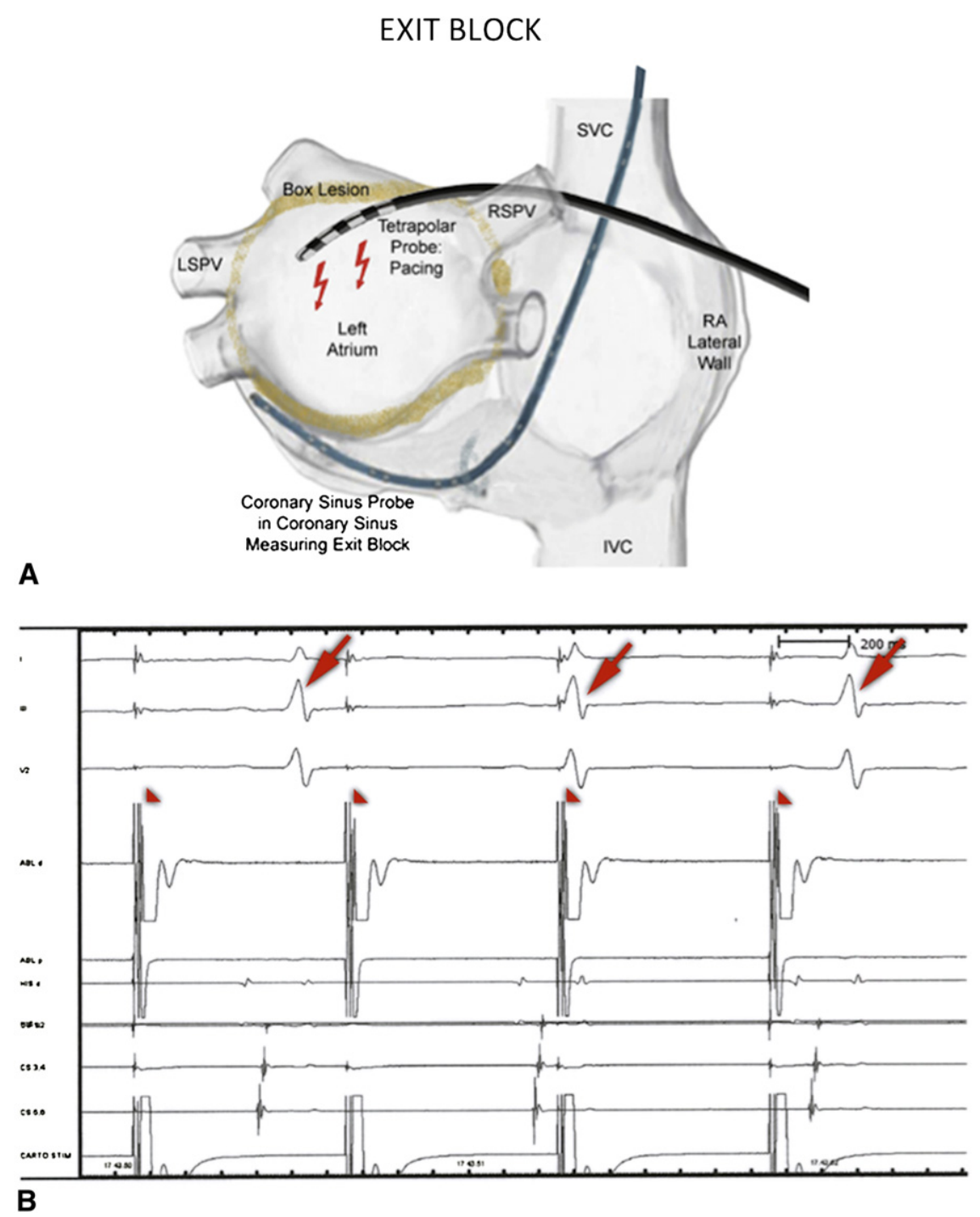

FIGURE 1. Schematic drawing (A) depicting a test of exit conduction block by means of pacing within the box lesion and sensing at the level of the coronary sinus. B, Absence of correlation between the pacing stimuli at the level of the pulmonary veins (arrowheads) and the surface electrocardiogram (arrows). SVC, Superior vena cava; $I V C$, inferior vena cava; $R S P V$, right superior pulmonary vein; $L S P V$, left superior pulmonary vein; $R A$, right atrium.

of the pericardial reflection to the oblique sinus. Moreover, from the EP standpoint, the presence of a common ostial trunk may represent a technical challenge in several instances that could be easily overcome by a surgical box lesion set.

The collaboration with EPs is extremely important for surgeons also in terms of intraoperative validation of the surgical ablation, thanks to the use of EP tools and end points. In our experience, it has consistently improved the quality of epicardial unipolar radiofrequency ablation, despite the potential concern in terms of efficacy of such an approach when achieving an effective conduction block. Instead, the intraoperative EP assessment allowed for a tailored delivery of radiofrequency applications, until at least entrance and/or exit block was demonstrated, as per HRS recommendations. ${ }^{8}$ As reported, we could achieve bidirectional block in $88.8 \%$ of patients and exit block in $100 \%$ of patients.

The interdisciplinary collaboration is also beneficial for EPs. Even though the current study did not aim at comparing the hybrid approach with other techniques (eg, transcatheter ablation alone), there is also a potential advantage in terms of reduced fluoroscopy and overall procedural time.

The treatment of the left atrial appendage by means of exclusion/excision is one of the cornerstones of the maze procedure and is associated with a reduced incidence of late thromboembolic events. To date, there are no safe and widely validated techniques and technologies for exclusion of the left atrial appendage via a right monolateral 


\section{ENTRANCE BLOCK}

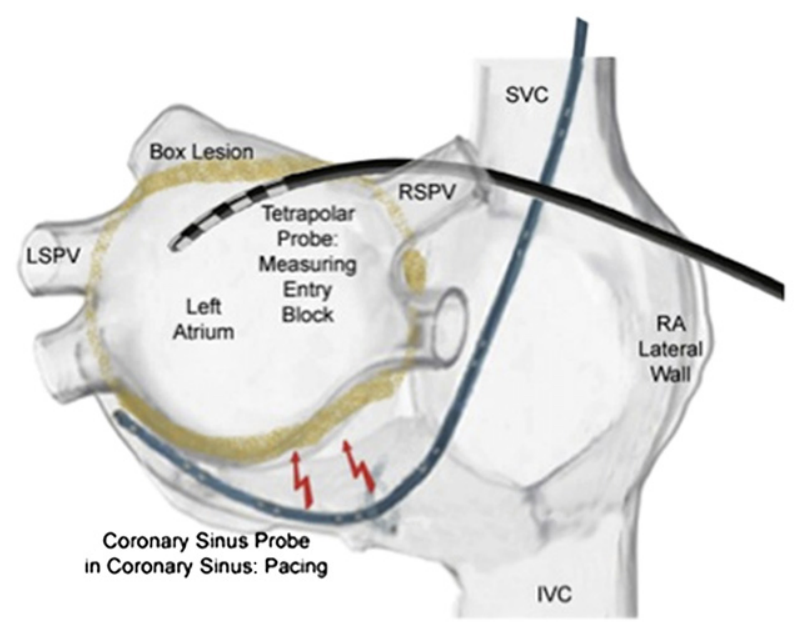

A

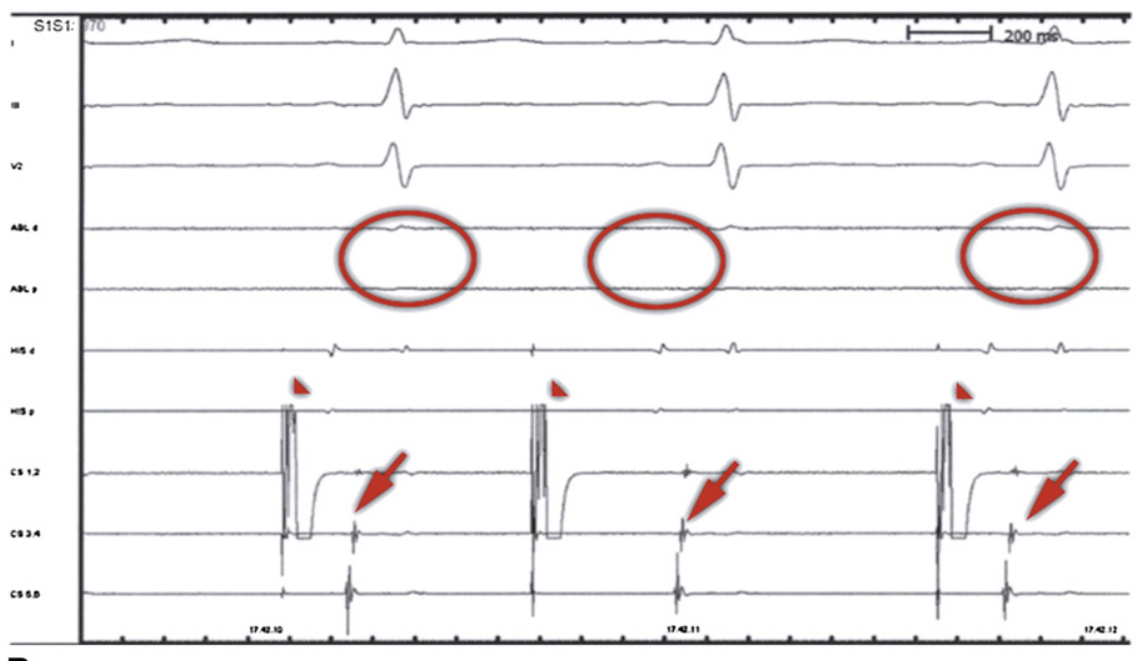

B

FIGURE 2. Schematic drawing (A) depicting test of entrance conduction block by means of pacing at the level of the coronary sinus and sensing within the box. B, Pacing at the level of the coronary sinus (arrowheads) with local capture (arrows) albeit without any recorded potentials within the box lesion (circles). SVC, Superior vena cava; $I V C$, inferior vena cava; $R S P V$, right superior pulmonary vein; $L S P V$, left superior pulmonary vein; $R A$, right atrium.

approach, and we therefore avoided any left atrial appendage treatment. Obviously, the possibility to continuously monitor the heart rhythm thanks to the implantable loop recorder allowed for a safe withdrawal of anticoagulation therapy in patients with a low $\mathrm{CHAD}_{2}-\mathrm{VASc}_{2}$ score and stable sinus rhythm.

In our experience, the possibility to delay the EP assessment from the surgical procedure allowed us to achieve important insights. First, it allowed for further "evolution" of the ablative lesions (usually occurring within a few weeks) to avoid false positive results in terms of early inducible arrhythmias. ${ }^{17}$ Then, false negatives may be minimized inasmuch as acute demonstration of a bidirectional block could only be transient and not confirmed by delayed testing at
1 month after surgery. In our series, for example, 2 patients "lost" acute bidirectional block while showing only exit block during the staged EP evaluation. Moreover, it was possible to perform tailored additional endocardial ablations (as depicted in the Results), which could have accounted for the excellent results even at 30 months' follow-up time.

Finally, the use of an implantable loop recorder allowed for continuous monitoring of heart rhythm and overcame the potential bias and drawbacks of spot or Holter electrocardiograms or even transtelephonic monitoring.

Inasmuch as a consistent percentage of patients (around $40 \%$ ) did not require any additional touch-up during the second EP evaluation, this subgroup of patients may have 
undergone unnecessary EP procedures after the surgical ablation. Nevertheless, the lack of clinical data with this approach led us to have all patients undergoing the sequential staged EP evaluation regardless of the rhythm outcome (with the implantable loop recorder). In fact, this approach allowed for an evaluation of the effectiveness of the surgical ablation in our preliminary series of hybrid ablations. We believe the protocol may be further adapted as more data are gathered, especially in the presence of a continuously stable sinus rhythm after the surgical ablation, which could therefore avoid the need for an additional EP evaluation.

The current study yields several limitations, especially in terms of the limited number of patients enrolled, and further evaluation of the sequential staged surgical-EP approach on a larger series of patients with a longer follow-up is warranted. Nevertheless, the current study proved the safety, feasibility, and preliminary efficacy of a novel therapeutic strategy for the treatment of P-AF and LSP-AF.

\section{References}

1. Krul SP, Driessen AH, Zwinderman AH, van Boven WJ, Wilde AA, de Bakker JM, et al. Navigating the mini-maze: systematic review of the first results and progress of minimally invasive surgery in the treatment of atrial fibrillation. Int J Cardiol. 2011 Nov 9 [Epub ahead of print].

2. Saltman AE, Gillinov AM. Surgical approaches for atrial fibrillation. Cardiol Clin. 2009;27:179-88

3. Mack MJ. Current results of minimally invasive surgical ablation for isolated atrial fibrillation. Heart Rhythm. 2009;6:S46-9.

4. Shen J, Bailey M, Damiano RJ Jr. Surgery for lone atrial fibrillation: present state-of-the-art. Innovations (Phila). 2009;4:248-55.

5. Weerasooriya R, Khairy P, Litalien J, Macle L, Hocini M, Sacher F, et al. Catheter ablation for atrial fibrillation: are results maintained at 5 years of follow-up? J Am Coll Cardiol. 2011;57:160-6.

6. Kabra R, Singh JP. Catheter ablation targeting complex fractionated atrial electrograms for the control of atrial fibrillation. Curr Opin Cardiol. 2012;27:49-54.

7. Bisleri G, Curnis A, Bottio T, Mascioli G, Muneretto C. The need of a hybrid approach for the treatment of atrial fibrillation. Heart Surg Forum. 2005;8:E326-30.

8. Calkins H, Kuck KH, Cappato R, Brugada J, Camm AJ, Chen SA, et al. 2012 HRS/EHRA/ECAS expert consensus statement on catheter and surgical ablation of atrial fibrillation: recommendations for patient selection, procedural techniques, patient management and follow-up, definitions, endpoints, and research trial design. Heart Rhythm. 2012;9:632-96.

9. Bisleri G, Muneretto C. Innovative monolateral approach for closed-chest atrial fibrillation surgery. Ann Thorac Surg. 2005;80:e22-5.
10. Koyama T, Tada H, Sekiguchi Y, Yamasaki H, Kuroki K, Mchino T, et al. Preven tion of atrial fibrillation recurrence with corticosteroids after radiofrequency catheter ablation: a randomized controlled trial. J Am Coll Cardiol. 2010;56:1463-72.

11. Botto GL, Padeletti L, Santini M, Capucci A, Gulizia M, Zolezzi F, et al. Presence and duration of atrial fibrillation detected by continuous monitoring: crucial implications for the risk of thromboembolic events. J Cardiovasc Electrophysiol. 2009;20:241-8.

12. Pokushalov E, Romanov A, Corbucci G, Artyomenko S, Turov A, Shirokova N, et al. Ablation of paroxysmal and persistent atrial fibrillation: 1-year follow-up through continuous subcutaneous monitoring. J Cardiovasc Electrophysiol. 2011;22:369-75.

13. Krul SP, Driessen AH, van Boven WJ, Linnenbank AC, Geuzebroek GS, Jackman WM, et al. Thoracoscopic video-assisted pulmonary vein antrum isolation, ganglionated plexus ablation, and periprocedural confirmation of ablation lesions: first results of a hybrid surgical-electrophysiological approach for atrial fibrillation. Circ Arrhythm Electrophysiol. 2011;4:262-70.

14. Mahapatra S, LaPar DJ, Kamath S, Payne J, Bilchick KC, Mangrum JM, et al Initial experience of sequential surgical epicardial-catheter endocardial ablation for persistent and long-standing persistent atrial fibrillation with long-term follow-up. Ann Thorac Surg. 2011;91:1890-8.

15. Kiser AC, Landers M, Horton R, Hume A, Natale A, Gersak B. The convergen procedure: a multidisciplinary atrial fibrillation treatment. Heart Surg Forum. 2010;13:E317-21.

16. Guiot A, Savouré A, Godin B, Anselme F. Collateral nervous damages after cryoballoon pulmonary vein isolation. J Cardiovasc Electrophysiol. 2011 Nov 14 [Epub ahead of print].

17. Magnano AR, Argenziano M, Dizon JM, Vigilance D, Williams M, Yegen H, et al. Mechanisms of atrial tachyarrhythmias following surgical atrial fibrillation ablation. J Cardiovasc Electrophysiol. 2006;17:366-73.

\section{Discussion}

Dr Charles R. Bridges (Charlotte, NC). I have a couple of questions. Could you provide more details of exactly how sinus rhythm was documented at 30 months and at each of the individual time points? What modality did you use? As you know, electrocardiography, for example, will certainly miss a large percentage of patients who have ongoing paroxysmal AF.

Dr Muneretto. Thank you for your question. As I showed in the slides, all patients received a continuous monitoring loop recorder. We analyzed the rhythms as a continuous phase for the entire follow-up period by 1 month, 3 months, 6 months, 9 months, 12 months, and every 3 months. I think that our results should be evaluated also according to that, because until now, the majority of papers published in the literature use spot electrocardiograms or spot Holter monitoring to assess recurrences, and obviously the use of continuous monitoring systems is much more specific for that. 